IdeAs

Idées d'Amériques

$18 \mid 2021$

Frontières dans les Amériques - Intégration, sécurité et migrations

\title{
Fronteras en las Américas - Integración, seguridad y migración: enfoques críticos
}

Pierre-Alexandre Beylier, Anne-Laure Amilhat-Szary, Gregory Benedetti y Éric Tabuteau

Traductor: Marta Gómez

\section{OpenEdition}

\section{Journals}

Edición electrónica

URL: https://journals.openedition.org/ideas/12002

DOI: $10.4000 /$ ideas. 12002

ISSN: 1950-5701

Editor

Institut des Amériques

Referencia electrónica

Pierre-Alexandre Beylier, Anne-Laure Amilhat-Szary, Gregory Benedetti y Éric Tabuteau, «Fronteras en las Américas - Integración, seguridad y migración: enfoques críticos», IdeAs [En línea], 18 | 2021,

Publicado el 01 octubre 2021, consultado el 11 noviembre 2021. URL: http://journals.openedition.org/ ideas/12002 ; DOl: https://doi.org/10.4000/ideas.12002

Este documento fue generado automáticamente el 11 noviembre 2021.

\section{(ब) $\Theta \Theta$}

IdeAs - Idées d'Amériques est mis à disposition selon les termes de la licence Creative Commons Attribution - Pas d'Utilisation Commerciale - Pas de Modification 4.0 International. 


\title{
Fronteras en las Américas - Integración, seguridad y migración: enfoques críticos
}

\author{
Pierre-Alexandre Beylier, Anne-Laure Amilhat-Szary, Gregory Benedetti y \\ Éric Tabuteau
}

Tradución : Marta Gómez

\section{Fronteras de laboratorio}

1 Las Américas constituyen un laboratorio de primer orden para estudiar el fenómeno de las fronteras y su evolución. Y no es solo porque, durante la década del «mundo sin fronteras», el continente americano ha visto cómo se han multiplicado los proyectos de integración regional -en particular con el MERCOSUR y el TLCAN, que han visto cómo los países se han acercado y han rebajado sus barreras aduaneras-, sino también porque, desde hace algunos años, esas mismas fronteras se han convertido en puntos de crispación debido a una serie de problemas que se han agravado, entre otros, tráficos de todo tipo y tensiones territoriales. Esta evolución reciente nos lleva a proponer una lectura crítica de los procesos fronterizos que recorren el continente para caracterizarlos mejor, tanto desde el punto de vista de sus especificidades continentales como de la manera en que esta región hace legibles las grandes tendencias mundiales. En un contexto mundial de auge teórico de los estudios sobre fronteras, puede ser interesante preguntarse en qué medida un enfoque continental permite evaluar las especificidades regionales, pero también contribuir de forma original a este esfuerzo epistemológico (Mezzadra S. y B. Neilson, 2013), (Nail, 2016), (Parker et al, 2012), (WastlWalter, 2012).

2 En este número de la revista IdeAs hemos querido abordar la temática de las fronteras a través del prisma de las dos dinámicas - a primera vista antitéticas- de la seguridad y la integración, así como de su impacto en la migración. Presentes en procesos contradictorios de «desmantelamiento y construcción» (Machado De Olivera, 2009: 19), 
estas dinámicas no solo estructuran fronteras -tanto reticulares como lineales- que sirven para ordenar los flujos de la globalización (Amilhat-Szary A.L., 2015), sino que también entran en colisión, lo cual, in fine, refuerza la ambivalencia de esta «institución» denominada frontera.

El continente americano ha sido escenario del apetito colonial de los poderes europeos y se ha visto sometido a su influencia directa durante casi cuatro siglos, desde su (re)descubrimiento en 1492 hasta la descolonización en la primera mitad del siglo xIx. Espacio de creación de zonas de influencia y de implantación de auténticos imperios coloniales, las Américas se convirtieron en una prolongación del Viejo Mundo y, al ser una fuente inagotable de recursos, en una pieza clave de la economía mundializada que empezó a desarrollarse después. Desde la descolonización, el continente se ha convertido en un espacio en donde Occidente reinventa constantemente las modalidades de su liderazgo global [Rouquié A., 1987].

4 Un aspecto que tienen en común las Américas es que sus fronteras se fijaron con el fin de «ordenar» el continente (Popescu G., 2011), antes incluso de que se crearan los Estados-nación. Más concretamente, estas fronteras combinan de manera original dos formas de apropiación territorial: por una parte, una lógica de conquista zonal colonizadora (frontier), cuyo objetivo es poner en valor un entorno considerado salvaje (wilderness) -lógica que fue teorizada por Frederick Turner en lo que respecta a Estados Unidos (Turner F., 1964) - y, por otra parte, el deseo de definir un mundo en redes desde la perspectiva occidental del espacio (boundary) (Perrier Bruslé L., 2007).

Esta doble lógica de conquista se aplicó en detrimento de las poblaciones amerindias que poblaban el continente. Los poderes coloniales, y luego los Estados recién independizados, se han servido de las fronteras - sistema de organización espacial ajeno a las poblaciones autóctonas- para esclavizarlas y dominarlas limitando su movilidad, condenándolas a señalar residencia en reservas o en algunos países, o imponiéndoles una identidad «nacional» que no era la suya (Rensink B., 2018). La «violencia» de las fronteras (Jones $\mathrm{R}, 2017$ ) no es nueva $\mathrm{y}$, en lo que se refiere a las Américas, su carácter sobreimpuesto y exógeno, cuando algunos las verían como antecedentes, enraíza esta violencia en la historia misma del continente, planteando preguntas en términos de legitimidad e identidad.

6 Más allá de las características históricas que les son propias, las fronteras americanas tienen características comunes a las del resto del mundo. En un contexto general, marcado por el auge de la globalización y las repercusiones de los atentados del 11 de septiembre de 2001, las fronteras están sometidas a fenómenos a priori antitéticos, y sin embargo concomitantes, de debordering y rebordering (Herzog L. y C. Sohn, 2019): por un lado, se borran y se desfuncionalizan para facilitar el paso de los flujos comerciales y financieros $\mathrm{y}$, por otro, se refuerzan para controlar movilidades de todo tipo y estigmatizar determinado tipo de migración. Esto es característico de un fenómeno que establece distintas formas de cruzar una misma frontera en función de una combinación de criterios que individualizan y definen «fronteridades» o «borderities» muy dispares (Amilhat Szary, A.-L. y Giraut F., 2015).

7 Su aparente desfuncionalización (Eva F., 1996) cobró impulso en los años 1990 cuando algunos países, abrazando las fuerzas de la globalización, establecieron diversos acuerdos comerciales (el TLCAN en América del Norte, el MERCOSUR en América del Sur, o la UNASUR, a escala del hemisferio sur). Con el objetivo de favorecer la integración regional, estos acuerdos pusieron de relieve una visión muy particular de la 
frontera, que se presentaba más como un «recurso» que como un «estigma» (AmilhatSzary, A.L., 2015: 85). La integración también se ha considerado un «factor de estabilización» (Medina L., 2009: 41) en el continente, en el cual algunas regiones han estado marcadas por recurrentes conflictos fronterizos desde el siglo xIX y donde ciertas fronteras siguen siendo objeto de disputa todavía hoy (Medina L., 2009: 36-37).

Con todo, a pesar de haberse abierto a los beneficios de los intercambios y de la economía de mercado, las fronteras americanas han visto cómo se han reforzado sus funciones. Desde los atentados del 11 de septiembre de 2001, la amenaza del terrorismo internacional ha empujado a los países del continente, con Estados Unidos a la cabeza, a reconsiderar el papel de sus límites internacionales. Con el fin de asegurar los territorios, las fronteras se han convertido en un punto de vigilancia y control hightech, en cuya convergencia pueden llegar a erigirse verdaderos muros como reflejo de fenómenos de refronterización y de la violencia que llevan asociada. Algunas fronteras que antes prácticamente no estaban vigiladas - por ejemplo, la frontera entre Canadá y Estados Unidos o las díadas latinoamericanas- han visto desplegarse en ellas toda una serie de medidas nuevas, desde drones hasta sensores terrestres, pasando por torres equipadas con cámaras de visión nocturna (Beylier P.A., 2016). Aunque la lucha contra el terrorismo domina los discursos, parece también que se utiliza como pretexto para controlar la migración, un fenómeno que actualmente es el objetivo explícito de esas medidas. La crisis ligada a la COVID-19, durante la cual vimos cómo se cerraba una frontera tras otra, ha dado a este fenómeno una magnitud sin precedentes. El texto de Nicolli Bernardes Ribeiro aborda esta perspectiva en las fronteras de Brasil, donde se plasma la contradicción entre el discurso nacionalista y un largo periodo de rechazo al cierre por razón de la pandemia.

9 Así pues, es desde esta perspectiva continental desde donde esta colección de artículos examina la propia noción de frontera y pone de relieve la problemática definición del concepto. El artículo de Jean Clot sobre las «fronteras porosas» ilustra la adaptación del discurso a este contexto cambiante. De forma complementaria, François-Michel Le Tourneau resalta la «lógica reticular» de los buscadores de oro de la Guayana Francesa que aprovechan la falta de vigilancia en zonas fronterizas remotas, yendo así en contra de la «lógica a-real» de los Estados para los cuales las fronteras siguen siendo herramientas de soberanía y vigilancia. Las fronteras, ya sean contestadas, transgredidas, trascendidas, reforzadas o integradas, están así pues en el centro del debate político en las Américas. En el debate público, las fronteras parecen prevalecer ante los discursos que promueven la integración continental dentro de la TLCAN y su sucesor, el T-MEC, así como del MERCOSUR, pero también a escala de las dos Américas (UNASUR) que constituyen conjuntos regionales que en los años 90 se presentaban como modelos.

No obstante, el «trabajo de la frontera» (traduciendo la expresión en inglés border work) no es, ni en América ni en otros lugares, una prerrogativa de actores dominantes, sino que es el fruto de prácticas «desde abajo» (Rumford C., 2008). En este sentido, algunos artículos se centran en la manera en la que los habitantes de las regiones fronterizas se relacionan con las normas internacionales con las que conviven y a las que desafían, en particular en el caso de la minería a pequeña escala en la Guayana Francesa, la cual parece emanciparse de esas normas y funcionar de forma «a-nacional», tal y como ilustra el texto de François-Michel Le Tourneau. La frontera, que es una «marca de identidad» (Piermay J.L., 2005: 206), pone de relieve la cuestión del vínculo entre la 
identidad, el territorio y las fronteras. Esta cuestión es tanto más importante cuanto que, en el continente, «el Estado ha precedido a la nación» (Medina L., 2009: 38). El papel atribuido a las fronteras en la «cohesión nacional» (Ibid) parece ser tanto más importante cuanto que puede entrar en conflicto con las representaciones de las comunidades fronterizas en las cuales la interpretación que las poblaciones autóctonas hacen de la construcción de las fronteras puede tener un papel esencial (Nates Cruz B., 2013). En los textos reunidos en este número se destacan las negociaciones y los enfrentamientos entre las poblaciones regionales y las poblaciones móviles presentes en las regiones fronterizas, siendo percibida la cuestión migratoria como un problema clave en los espacios fronterizos americanos hoy día.

\section{Seguridad y migración en las fronteras de las Américas: ¿dos términos necesariamente relacionados?}

Los atentados del 11 de septiembre de 2001 -y, en general, la aparición de una amenaza terrorista internacional, presente en América Latina desde los atentados de Buenos Aires en los años 80- han modificado el papel de las fronteras. El surgimiento de una Fortress America (Alden E., 2008; Andreas P., 2003; Noble J., 2004) ha sido ampliamente documentado en lo que se refiere a los Estados Unidos, pero el fenómeno del rebordering atañe también a las fronteras latinoamericanas. En este sentido, podemos adivinar en el continente una política de reinterpretación original de las grandes tendencias de la gestión de fronteras a nivel mundial, por ejemplo, con el despliegue de un aparato de seguridad en las fronteras brasileñas a una escala sin precedentes, sin que exista un cuestionamiento real del aumento de los flujos de intercambios internacionales, tanto legales como ilegales (contrabando, narcotráfico, etc.) (Dorfman A. et al, 2014; Dorfman A. et al, 2017).

12 El artículo de Beatriz Zepeda sobre el trabajo de los funcionarios de seguridad en la frontera guatelco-mexicana, o el texto de Cléa Fortuné sobre la interpretación de la seguridad que hace la comunidad transfronteriza de Douglas (Arizona) y Agua Prieta (Sonora) dan una visión matizada del trabajo humano y de las negociaciones que en el día a día construyen el gran paradigma de la «seguridad». Hay, así pues, un interés por los aspectos materiales de una dinámica de este tipo y por la manera en que este proceso se territorializa. Los enfoques históricos que renuevan la cuestión del conflicto fronterizo territorializado y multiplican las escalas de lectura, permiten en este contexto la evolución de las narrativas nacionales y nacionalistas.

13 Las diferentes políticas aplicadas desde principios de los años 2000 en las Américas ilustran de manera particular el fenómeno del rebordering que se está jugando a escala mundial. Los artículos reunidos aquí examinan tanto los dispositivos como sus implicaciones para las relaciones transfronterizas, dentro de un enfoque comparativo (cf. artículo de Jean Clot o de Laurence Cros) que permite no solo poner de manifiesto las dinámicas que recorren América del Norte, sino también vincular las dos mitades de América... (Brunet-Jailly E., 2007; Konrad V. et al, 2008).

14 A pesar de los fenómenos comunes que las estructuran, tomadas individualmente, las fronteras americanas presentan una cierta especificidad, pues su función cambia de un país a otro e incluso de una región a otra (Machado Oliveira T. C., 2009: 20). En algunos 
casos, por el contrario, las fronteras están marcadas por una «inmensa variedad», en particular en América Latina, donde son más numerosas. Entre las «fronteras distantes» que separan a regiones marginales cuyos territorios «dan la espalda a la frontera» (Argentina/Chile, Paraguay/Brasil), las «fronteras serpenteantes», marcadas por vínculos transfronterizos ilegales, en particular en zonas recientemente urbanizadas (Costa Rica/Nicaragua, México/Guatemala), o incluso las «fronteras vibrantes», cuyo dinamismo viene dado por una densa población y numerosas ventajas comparativas (Brasil/Uruguay, Perú/Ecuador, México/Estados Unidos), sin olvidar las «fronteras protocolarias», que son regiones instrumentalizadas por el poder central para promover su «dinamización» o incluso luchar contra los tráficos ilegales según un enfoque top-down (Chile/Argentina, Haití/República Dominicana), vemos cómo existen numerosos tipos de fronteras (Machado de Oliveira T.C., 2009: 28-30). A través de ellas se forjan distintos grados de cooperación transfronteriza, dando lugar a un auge de las relaciones interterritoriales consideradas paradiplomáticas (González Miranda, S. et al., 2016).

Los cruces fronterizos y su creciente coste humano son elementos esenciales hoy día para entender las fronteras americanas (De Leon J. y M. Wells, 2015). Diversos artículos del dossier (concretamente, los de Marie-Laure Coubès y Jean Clot) muestran de forma llamativa hasta qué punto el paso de la línea fronteriza solo puede entenderse a diversas escalas. Efectivamente, hay que analizar tanto los flujos intracontinentales, vinculados sobre todo con la movilidad del trabajo, como la forma en que las Américas se enmarcan en estrategias migratorias a gran escala, con cada vez más personas que, por ejemplo, intentan llegar a América del Norte desde África, atravesando el Atlántico por la vieja ruta de los esclavos, y luego probando suerte tomando largas y peligrosas rutas hacia el norte. Todo ello pone de relieve cuestiones como la vulnerabilidad y el riesgo, además de las estrategias adoptadas por las comunidades fronterizas para convivir con la inseguridad (véase el texto de Clea Fortuné) y reinterpretar a su manera los mandatos internacionales. Los fenómenos ilegales que se han desarrollado en las Américas (el narcotráfico, la inmigración ilegal, los cárteles, etc.) no pueden abordarse sin profundizar en sus causas, sus ramificaciones, sus implicaciones para las poblaciones locales, así como las políticas creadas para combatirlas. Todo ello no debe hacernos olvidar las múltiples maneras en que las poblaciones conviven con las fronteras en el día a día, principalmente en temas de educación o de salud (véanse los artículos de Elisabeth Fauquert o de Isabelle Sinic).

Por último, al ser los espacios fronterizos lugares en constante evolución cuya expresión estética e imaginarios se recomponen rápidamente (Rodney L., 2017; Amilhat-Szary A.-L., 2014), la mediación artística del imaginario de la línea se presenta como un punto de entrada esencial para entender la evolución en curso de las fronteras americanas. El texto de Marion Gautreau dedicado al enfoque fotográfico de la frontera entre México y Estados Unidos, analizada de forma retrospectiva a lo largo de más de cuarenta años a través del trabajo de Elsa Medina y de Francisco Mata Rosa, responde a esta búsqueda. 


\section{La lógica en la organización del número 18 de la revista}

17 Los artículos que se presentan en este dossier abordan la problemática común de las fronteras de tres formas complementarias. Tres de ellos estudian la definición del concepto de seguridad, demostrando su aspecto a la vez multiforme y multiescalar, por una parte, y la importancia de la interpretación que los actores locales hacen de los desafíos mundiales, por otra. Es el caso del artículo de Beatriz Zepeda titulado «La seguridad en la frontera entre México y Guatemala. Entender los riesgos y amenazas desde el punto de vista de los responsables de la seguridad mexicanos y guatemaltecos», del artículo de François-Michel Le Tourneau, «¿La frontera? ¿Qué frontera? Dinámica transnacional de la minería ilegal de oro en la Guayana Francesa», así como del texto de Nicolli Bernardes Ribeiro «Contingencia pandémica e impactos migratorios: análisis de los actos normativos para cerrar la frontera entre Brasil y Venezuela».

Una segunda serie de textos se interesa por la cuestión migratoria planteando el complejo vínculo entre la migración y los problemas de seguridad. Entran aquí los artículos de Marie-Laure Coubès «De los trabajadores mexicanos indocumentados a las familias centroamericanas solicitantes de asilo: la transformación de los flujos migratorios en la frontera entre México y Estados Unidos, 2005-2020)», el de Laurence Cros, «Solicitudes de asilo en la frontera entre Canadá y Estados Unidos: el acuerdo entre ambos países sobre terceros países seguros y los valores canadienses», y, por último, el texto de Jean Clot sobre «La frontera entre México y Guatemala: ¿una frontera "porosa"?».

El último grupo de artículos aporta un estimulante matiz a la oposición, a menudo caricaturizada, entre seguridad y migración, mostrando cómo esta última se negocia a diario mediante prácticas que renuevan sus términos. Lo que demuestra Cléa Fortuné de forma global a escala de una aglomeración en «Tensiones en la seguridad fronteriza: entre la seguridad del Estado y la inseguridad económica local» se presenta a través de las prácticas escolares en las fronteras en el texto de Isabelle Sinic "Cooperación educativa entre Estados Unidos y México: qué perspectivas para la educación de los estudiantes transnacionales», o médicos, a través del artículo de Elisabeth Fauquert, "Sometimes the treatment you seek is just one flight away: causas y límites de la nueva movilidad sanitaria en Estados Unidos en el siglo xXI». Por último, en «Miradas sobre la línea. La frontera México-Estados Unidos a través del objetivo de Elsa Medina y Francisco Mata Rosas», la autora Marion Gautreau nos ofrece un texto cuyo enfoque histórico confirma los análisis contemporáneos y cuya dimensión artística los prolonga.

\section{Aperturas}

Más allá de la visión de conjunto que ofrecen los artículos reunidos en este número, quedan pendientes de profundizar un cierto número de temas para comprender mejor la evolución de las fronteras americanas. La cuestión de las fronteras marítimas, cuya delimitación provoca grandes tensiones, sobre todo en Centroamérica (Medina L., 2009: 40 y Medina L., 2021), debería estar mejor documentada dentro del continente. Este tema de las fronteras marítimas abre también la reflexión acerca de las fronteras 
externas del continente, especialmente en el frente ártico (Nicol H. et al, 2009) y, de forma más genérica, plantea la cuestión de la dimensión medioambiental de las cuestiones transfronterizas (Guyot S., 2011; Wadewitz L., 2012), que adquiere una dimensión singular en las Américas, donde, en su mayoría, los límites internacionales atraviesan zonas de baja densidad y ocupación humana.

Por último, cabe subrayar que el continente americano sigue salpicado por una miríada de fronteras que no son geopolíticas sino sobre todo sociales y raciales. La cuestión específica de las fronteras urbanas (Chevalier J. et al, 2004) en los espacios continentales que comparten la misma experiencia de desarrollo ligada al neoliberalismo económico plantea numerosas preguntas sobre su segmentación. Quedan por construir enfoques interseccionales para seguir explorando la multidimensionalidad de los procesos fronterizos en las Américas.

\section{BIBLIOGRAFÍA}

Alden, Edward, The Closing of the American Border, New York City, Harper and Collins, 2008.

Andreas, Peter et Thomas J. Biersteker, The Rebordering of North America: Intégration and Exclusion in a New Security Context, New York, Routledge, 2003.

Amilhat-Szary, Anne-Laure. 2014. « Latin American Borders on the Lookout: Recreating borders through art in the Mercosul ». P. 346-78 in Making the Border in Everyday Life, édité par R. Jones et C. Jonhson. Farnham, Ashgate.XAmilhat-Szary, Anne-Laure, Qu'est-ce qu'une frontière aujourd'hui ? 3e tirage. Paris, PUF, 2015.

Amilhat-Szary, Anne Laure, et Frédéric Giraut (dir.), Borderities: The Politics of Contemporary Mobile Borders, Basingstoke, Palgrave Macmillan, 2015.

Beylier, Pierre-Alexandre, Canada-États-Unis, les enjeux d'une frontière, Rennes, Presses universitaires de Rennes, Des Amériques, 2016

Brunet-Jailly, Emmanuel (dir.), Borderlands: Comparing Border Security in North America and Europe, Ottawa, University of Ottawa Press, 2007.

Chevalier, Jacques et Cristina Carballo, « Fermetures résidentielles et quête de l'entre-soi, entre Nord et Sud Des Amériques », L'espace Géographique, vol. 33, n4, 2004, p. 325-335.

De Leon, Jason, et Michael Wells, The Land of Open Graves, Los Angeles, University of California Press, 2015.

Dorfman Adriana, et Daniel Francisco de Bem, « Contrabando, Tragédia e Reflexividade: Antígona Na Fronteira Gaúcha », in Revista Do Centro de Educação e Letras, n¹5, 2014, p. 33-51.

Dorfman, Adriana et al., « Political Commodities and Sovereignty Management: Cigarette Smuggling across Brazil's Southern Borders », in Geopolitics, vol.22, n 4, 2017, p. 863-886 <https://doi.org/10.1080/14650045.2017.1356288>. 
Eva, Fabrizio, 1996, «International Boundaries, Geopolitics and the (Post)Modern Territorial Discourse: The Functional Fiction ». in Newman, David (dir.), Boundaries, Territory and Postmodernity, London, Taylor \& Francis, p. 32-51.

Gonzalez Miranda, Sergio, et al. (dir.), Relaciones transfronterizas y paradiplomacia en América Latina. Aspectos teóricos y estudio de casos, Santiago de Chile, RIL editores, 2016.

Guyot, Sylvain, « The Eco-Frontier Paradigm: Rethinking the Links between Space, Nature and Politics » in Geopolitics n²16(3), 2011, p.675-706.

Herzog, Lawrence A., et Christophe Sohn, « The Co-Mingling of Bordering Dynamics in the San Diego-Tijuana Cross-Border Metropolis » in Territory, Politics, Governance 7 (2): 2019, p. 177-99, https://doi.org/10.1080/21622671.2017.1323003.

Jones, Reece, Violent Borders: Refugees and the Right to Move, London/ New York, Verso, 2017.

Konrad, Victor, et Heather Nicol, Beyond Walls: Re-Inventing the Canada-United States Borderlands, London, Ashgate, 2008.

Machado de Oliveira, Tito Carlos, «Frontières en Amérique latine : réflexions méthodologiques ", Espaces et sociétés, vol. 138, no. 3, 2009, pp. 19-33.

Medina, Lucile, «Les frontières de l'isthme centraméricain, de marges symboliques à des espaces en construction ", in Espaces et sociétés vol. 138, no. 3, 2009, p. 35-50.

Medina, Lucile, « Pouvoir, préservation, prédation. Les frontières d'Amérique latine témoins d'un continent sous tensions » in L'Espace Politique. Revue en ligne de géographie politique et de géopolitique, $n^{\circ} 42,2021$.

Mezzadra, Sandro, et Brett Neilson, Border As Method, or, the Multiplication of Labor, Durham, NC, Duke University Press, 2013.

Nail, Thomas, Theory of the Border, Oxford/ New York, Oxford University Press, 2016.

Nates Cruz, Beatriz (dir.), La Frontera, Las Fronteras. Diálogos Transversales En Estudios Territoriales Contemporáneos, Editorial Universitaria de Caldas, 2013.

Nicol, Heather et Lassi Heininen, « Networking the North: Cross Border Connections and the New International Circumpolar Geopolitics » in Southern Journal of Canadian Studies, vol. 2009/2, 2009, p. 11-26.

Noble, John, « Fortress America or Fortress North America? », paper prepared for the IRPP on North American Integration: Migration, Trade and Security, April 2004.

Parker, Noel et Nick Vaughan-Williams, « Critical Border Studies: Broadening and Deepening the "Lines in the Sand" Agenda » in Geopolitics, vol. 17, 2012, p.727-33.

Perrier-Bruslé, Laetitia, « The Front and the Line: The Paradox of South American Frontiers Applied to the Bolivian Case » in Geopolitics, vol.12, 2007, p. 57-77.

Piermay, Jean-Luc, « La Frontière et ses ressources : regards croisés » in Antheaume, Benoît et Frédéric Giraut (dir.) Le territoire est mort : vive les territoires ! : une refabrication au nom $d u$ développement, Montpellier, IRD Editions, 2005, p. 2013-221.

Popescu, Gabriel, Bordering and Ordering the Twenty-first Century: Understanding Borders, Plymouth, Rowman and Littlefield Publishers Inc., 2011.

Rensink, Brenden, Native but Foreign, Indigenous Immigrants and Refugees in the North American Borderlands, College Station, TX, Texas A\&M University Press, 2018. 
Rodney, Lee, Looking beyond Borderlines: North America's Frontier Imagination, New York, Routledge, Taylor \& Francis Group, Routledge Advances in Art and Visual Studies \#19, 2017.

Rouquié, Alain, Amérique latine : introduction à l'Extrême-Occident, Paris, Seuil, 1987.

Rumford, Chris (dir.), Citizens and Borderwork in Contemporary Europe, New York/ London, Routledge, 2008.

Turner, Frederick. J., La Frontière dans l'histoire des États-Unis, Paris, Presses universitaires de France, 1963 [1893].

Wadewitz Lissa K., The Nature of Borders. Salmon, Boundaries, and Bandits on the Salish Sea, Bellingham, University of Washington Press, 2012.

Wastl-Walter, Doris (dir.), Companion to Border Studies, Farnham, Ashgate, 2012.

\section{AUTORES}

\section{PIERRE-ALEXANDRE BEYLIER}

Pierre-Alexandre Beylier est actuellement maître de conférences en études nord-américaines à l'université Grenoble-Alpes. Ancien élève de l'Ecole Normale Supérieure de Cachan et Agrégé d'anglais, il a effectué une thèse à l'Université Paris 3-Sorbonne Nouvelle, portant sur les mutations qu'a connues la frontière Canada/Etats-Unis depuis le 11 septembre 2001. Il a publié aux Presses Universitaires de Rennes un ouvrage sur ce sujet : Canada/Etats-Unis : les enjeux d'une frontière. Il s'intéresse désormais aux questions relatives aux communautés frontalières et aux villes-frontière.

\section{ANNE-LAURE AMILHAT-SZARY}

Anne-Laure Amilhat Szary est professeure à l'Université Grenoble-Alpes. Ancienne élève de l'ENS Fontenay, agrégée de Géographie et membre honoraire de l'IUF, c'est une géographe politique qui analyse les frontières. Elle développe des recherches concernent les interrelations entre espace et art dans les lieux contestés et construit un musée imaginaire du border art. Cofondatrice du collectif antiAtlas des frontières (http://www.antiatlas.net/), elle anime le Performance Lab dédié à la Recherche-Création.

Auteure de : Après les frontières, avec la frontière (2006, avec M.-C. Fourny), Qu'est-ce qu'une frontière aujourd'hui? (2015), Borderities, the Politics of Mobile Borders (2015, avec F. Giraut), Histoires de frontières, une enquête sud-africaine (2017, collectif), Géopolitique des frontières. Découper la terre, inventer une vision du monde (2020), Frontières (2020, avec G. Hamez)

\section{GREGORY BENEDETTI}

Gregory Benedetti, agrégé d'anglais et docteur en civilisation américaine, est maître de conférences à l'Université Grenoble Alpes, et membre de l'Institut des langues et des cultures d'Europe, Amérique, Afrique, Asie et Australie. Ses recherches portent essentiellement sur la question raciale aux États-Unis, et plus particulièrement sur la communauté afro-américaine, tant sur le plan politique que culturel. Ses premières publications s'intéressaient notamment à la nouvelle génération d'hommes politiques noirs, dont Barack Obama est devenue la figure tutélaire, mais non exclusive, en devenant président des États-Unis en 2008. Ses travaux s'intéressent également à l'histoire et la politique afro-américaine dans son ensemble, ainsi qu'à la représentation de la communauté noire dans les productions culturelles comme les séries télévisées. Il a dernièrement travaillé sur les élections présidentielles américaines en co- 
organisant une journée d'études à l'Université Grenoble Alpes. Il est membre de l'Association française d'études américaines depuis 2008.

\section{ÉRIC TABUTEAU}

Professeur des Universités, Université d'Orléans.

UFR des lettres, langues et arts. 